\title{
Black holes in New Massive Gravity dressed by a (non)minimally coupled scalar field
}

\author{
Francisco Correa* \\ Centro de Estudios Científicos (CECs), Valdivia, Chile. \\ Mokhtar Hassainđ开 \\ Instituto de Matemática y Física, Universidad de Talca, Casilla 747, Talca, Chile. \\ Julio Oliva \\ Instituto de Ciencias Físicas y Matemáticas, Universidad Austral de Chile, Valdivia, Chile
}

\begin{abstract}
We consider a self-interacting, massive scalar field (non)minimally coupled to new massive gravity in three dimensions. For this model, we first derive a family of black hole solutions depending on a unique integration constant and parameterized in terms of the nonminimal coupling parameter. Imposing the absence of naked singularities restricts the parameters in such a way that the field vanishes at infinity and fixes the metric to be asymptotically AdS. Within this family of solutions it is possible to find a black hole supported by a minimally coupled scalar field and therefore the existence of these solutions is not inherent to the presence of the nonminimal coupling. The Wald formula for the entropy, being proportional to the lapse function evaluated at the horizon, yields a zero entropy in spite of the fact that the solution has a non zero temperature. As a consequence, the unique integration constant may be interpreted as a sort of gravitational hair. As in the source free case, we show that the same field equations admit as well asymptotically Lifshitz black hole solutions in a different region of the space of parameters. These Lifshitz solutions are divided in three families for which all the parameters entering in the action may be expressed in term of the dynamical exponent.
\end{abstract}

\section{INTRODUCTION}

From the discovery of the BTZ black hole solution [1], there has been an increasing interest for black hole physics in three dimensions. This interest has taken much bigger proportion the last two decades in part because of their implication in the context of $\mathrm{AdS}_{3} / \mathrm{CFT}_{2}$ correspondence. Indeed, it is now well accepted that threedimensional gravity is an excellent laboratory in order to explore and test some of the ideas behind the AdS/CFT correspondence [2]. In contrast with the four-dimensional case, the existence of the BTZ black hole in three dimensions is inherent to the presence of the negative cosmological constant and due to the lack of local degrees of freedom in pure Einstein gravity in three dimensions it is the global structure of this solution what provides it's non-triviality. A way to circumvent this behavior was proposed long time ago in [3] by adding to the standard Einstein-Hilbert action a Chern-Simons term built out of the connection. In second order formulation, the resulting theory called topologically massive gravity which is not parity invariant because of the Chern-Simons term, propagates a single massive spin 2 (left or right) degree of freedom depending on the sign of the Chern-Simons coupling parameter. More recently, a massive gravity respecting parity invariance has been proposed in Ref. [4], where the authors showed that a particular and specific combination of the quadratic invariants constructed with the scalar curvature $R$ and the Ricci tensor $R_{\mu \nu}$ kill

\footnotetext{
*Electronic address: correa-at-cecs.cl

${ }^{\dagger}$ Electronic address: hassaine-at-inst-mat.utalca.cl

$\ddagger$ Electronic address: julio.oliva-at-uach.cl.
}

the dangerous spin 0 mode leaving only a healthy massive spin 2 field. Soon after its presentation, this theory whose action in presence of cosmological term $\Lambda$ is given by

$$
S_{N M G}=\frac{1}{2} \int d^{3} x \sqrt{-g}\left[R-2 \Lambda-\frac{1}{m^{2}}\left(R_{\mu \nu} R^{\mu \nu}-\frac{3}{8} R^{2}\right)\right],
$$

was dubbed new massive gravity. This new gravity theory has been intensively studied during the last years, and shown to admit interesting physical solutions as for examples AdS waves [5], warped AdS black hole [6], type $D$ and type $N$ solutions, see [7] and [8]. It is also interesting to stress that new massive gravity accommodates black hole solutions with rather different asymptotic behaviors. Indeed, within the black hole spectra of solutions of the theory, one finds rotating asymptotically AdS black holes with a gravitational hair [9] as well as a Lifshitz black hole with a dynamical exponent $z=3$ [10]. By Lifshitz black hole, we mean a black hole spacetime whose asymptotic behaviour enjoys of an anisotropic scaling symmetry where time and space scale with different weights. Recently, there has been an intense activity to promote the ideas underlying the gauge-gravity duality to non-relativistic physics with the hope of gaining a better understanding of some strongly coupled condensed matter physics phenomena observed in laboratories, for a review see e. g. [11, 12]. In this context, Lifshitz spacetimes whose metrics read

$$
d s_{\mathcal{L}}^{2}=-r^{2 z} d t^{2}+\frac{d r^{2}}{r^{2}}+r^{2} d \vec{x}_{D-2}^{2}
$$

are natural candidates to be the gravity duals for nonrelativistic scale invariant theories, [13]. Here, the dynamical exponent $z$ reflects the anisotropy of the scaling symmetry

$$
t \rightarrow \lambda^{z} t, \quad r \rightarrow \frac{r}{\lambda}, \quad \vec{x} \rightarrow \lambda \vec{x} .
$$


In the present work, we establish that new massive gravity in three dimensions may also accommodate black hole solutions with a source given by a (non)minimally coupled and self-interacting scalar field whose action reads

$$
S_{M}=-\int d^{3} x \sqrt{-g}\left[\frac{1}{2} \partial_{\mu} \Phi \partial^{\mu} \Phi+\frac{\xi}{2} R \Phi^{2}+\frac{M}{2} \Phi^{2}+\frac{\lambda}{4 !} \Phi^{4}\right]
$$

Here, $\xi \geq 0$ denotes the nonminimal coupling parameter, $R$ the scalar curvature, $M$ is a constant identified as part of the mass of the scalar field and $\lambda$ is the coupling constant of the potential $\Phi^{4}$. The field equations obtained by varying the action $S_{N M G}+S_{M}$ read

$$
\begin{aligned}
& G_{\mu \nu}+\Lambda g_{\mu \nu}-\frac{1}{2 m^{2}} K_{\mu \nu}=T_{\mu \nu}, \\
& \square \Phi=\xi R \Phi+M \Phi+\frac{\lambda}{3 !} \Phi^{3},
\end{aligned}
$$

where we have defined

$$
\begin{aligned}
K_{\mu \nu} & =2 \square R_{\mu \nu}-\frac{1}{2} \nabla_{\mu} \nabla_{\nu} R-\frac{1}{2} \square R g_{\mu \nu}+4 R_{\mu \alpha \nu \beta} R^{\alpha \beta} \\
& -\frac{3}{2} R R_{\mu \nu}-R_{\alpha \beta} R^{\alpha \beta} g_{\mu \nu}+\frac{3}{8} R^{2} g_{\mu \nu} .
\end{aligned}
$$

and the stress tensor is given by

$$
\begin{aligned}
T_{\mu \nu}= & \partial_{\mu} \Phi \partial_{\nu} \Phi-g_{\mu \nu}\left(\frac{1}{2} \partial_{\sigma} \Phi \partial^{\sigma} \Phi+\frac{M}{2} \Phi^{2}+\frac{\lambda}{4 !} \Phi^{4}\right) \\
& +\xi\left(g_{\mu \nu} \square-\nabla_{\mu} \nabla_{\nu}+G_{\mu \nu}\right) \Phi^{2} .
\end{aligned}
$$

In the case of pure Einstein gravity with a cosmological constant, there exists a broad literature of black hole solutions with a (non)minimal scalar field which started with the pioneering work of Martinez and Zanelli [14], see e. g. [15 18]. In this note, we revisit this problem in the context of new massive gravity. Interestingly enough, we will show that, as in the free source case, there exist a family of asymptotically AdS black hole solutions as well as Lifshitz black hole configurations.

The plan of the paper is the following. In the next section, we present a family of AdS black hole solution depending on a unique integration constant. For this class of solutions, the constants $m, M, \Lambda$ and $\lambda$ are all parametrized in terms of the nonminimal coupling parameter and the AdS radius $l$. We also show that these solutions may even exist for a scalar which is minimally coupled to gravity $(\xi=0)$, while if $\xi$ takes the value that provides a conformally coupled scalar $(\xi=1 / 8)$, the solution reduces to a stealth black hole configuration on a particular case of the asymptotically AdS black hole of [9]-223]. In Sec. 3, three classes of asymptotically Lifshitz black holes are reported for which all the parameters entering in the action are fixed in terms of the dynamical exponent. The last section is devoted to the discussion and some conclusions.

\section{ASYMPTOTICALLY ADS BLACK HOLE SOLUTIONS}

A black hole solution of the field equations (2) is given by

$$
\begin{aligned}
d s^{2} & =-F(r) d t^{2}+\frac{d r^{2}}{F(r)}+r^{2} d \varphi^{2}, \\
F(r) & =\frac{r^{2}}{l^{2}}-c_{1}\left(\frac{r}{l}\right)^{\frac{32 \xi-5}{16 \xi-3}}, \\
\Phi(r) & =\left(\frac{l}{r}\right)^{\frac{1}{6-32 \xi}} \sqrt{\frac{8 c_{1}(32 \xi-5)}{256 \xi^{2}-32 \xi-1}},
\end{aligned}
$$

where $c_{1}$ is an arbitrary integration constant, and the cosmological term in the action $\Lambda$, the self-interaction coupling $\lambda$, the graviton mass and the scalar field mass are fixed as

$$
\begin{aligned}
\Lambda & =-\frac{(16 \xi-1)(48 \xi-7)}{2\left(256 \xi^{2}-32 \xi-1\right) l^{2}} \\
\lambda & =-\frac{3(8 \xi-1)\left(256 \xi^{2}-32 \xi-1\right)\left(768 \xi^{2}-152 \xi+9\right)}{16(16 \xi-3)^{2}(32 \xi-5) l^{2}} \\
m^{2} & =\frac{256 \xi^{2}-32 \xi-1}{2(16 \xi-3)^{2} l^{2}} \\
M & =\frac{(8 \xi-1)\left(768 \xi^{2}-192 \xi+11\right)}{4(16 \xi-3)^{2} l^{2}}
\end{aligned}
$$

The isolated points defined by $\xi=\frac{1}{16}(1+\sqrt{2}), \xi=\frac{5}{32}$ and $\xi=\frac{3}{16}$ are excluded from this family.

Imposing the absence of naked singularities at infinity implies that the coupling $\xi$ must be restricted such that the leading term in the lapse should be $r^{2}$ (therefore fixing an asymptotically AdS behaviour). As a consequence, the scalar field vanishes at infinity. Requiring in addition the existence of an event horizon, finally implies that the range of physically allowed values of $\xi$ is

$$
\xi \in\left[0, \frac{1}{16}(1+\sqrt{2})[\cup] \frac{5}{32}, \frac{3}{16}[,\right.
$$

Clearly the strenght of the subleading term in the metric strongly depends on the value of the nonminimal coupling parameter $\xi$.

The horizon is located at $r=r_{+}=l c_{1}^{3-16 \xi}$ while the Hawking temperature of these solutions is given by

$$
T_{H}=\frac{c_{1}^{3-16 \xi}}{4 \pi l(3-16 \xi)} .
$$

The Wald formula for the entropy $\mathcal{S}$, being proportional to the lapse metric function evaluated at the horizon yields a zero entropy

$$
\left.\mathcal{S} \propto\left(1-c_{1}\left(\frac{r}{l}\right)^{\frac{1}{16 \xi-3}}\right)\right|_{r=r_{+}}=0 .
$$

Note that zero entropy black hole solutions with planar horizon have also been found for scalar fields nonminimally coupled with the general Lovelock gravity in arbitrary dimension in [19]. Assuming that the first law of thermodynamics holds, implies that the solutions in our family have zero mass. As a consequence, one may interpret the unique integration constant $c_{1}$ as a gravitational hair.

To conclude this section, let us analyze in more detail the solutions obtained for some relevant particular values of $\xi$. 
It is remarkable to note that we are allowed to consider the minimally coupled case $(\xi=0)$, and therefore conclude that the existence of these solutions is not inherent to the presence of the nonminimal coupling $R \Phi^{2}$ in the action as it is the case for example in four dimensions, $20-22]$. For this case the metric reduces to

$$
d s^{2}=-\left(\frac{r^{2}}{l^{2}}-c_{1} \frac{r^{5 / 3}}{l^{5 / 3}}\right) d t^{2}+\frac{d r^{2}}{\frac{r^{2}}{l^{2}}-c_{1} \frac{r^{5 / 3}}{l^{5 / 3}}}+r^{2} d \phi^{2},
$$

and the values for the couplings can be read from equation (77) while the expression for the field is obtained from (6c) by fixing $\xi=0$.

When $\xi=\frac{1}{8}$, the scalar field becomes massless $M=0$ and not self-interacting $\lambda=0$, giving indeed a conformal invariant matter source. The remaining parameters take the values $\Lambda=m^{2}=-\frac{1}{2}$ while the metric reduces to

$$
d s^{2}=-\left(\frac{r^{2}}{l^{2}}-c_{1} \frac{r}{l}\right) d t^{2}+\frac{d r^{2}}{\frac{r^{2}}{l^{2}}-c_{1} \frac{r}{l}}+r^{2} d \phi^{2} .
$$

The expresion for the field can be read as well from (6c) by setting $\xi=\frac{1}{8}$. This solution is a particular case of the asymptotically AdS black hole found in the absence of sources for new massive gravity [9, 23]. In other words, for the conformal coupling $\xi=\frac{1}{8}$, the black hole becomes a particular solution of the field equations (2) for which both sides (the gravity and the source parts) vanish identically

$$
G_{\mu \nu}+\Lambda g_{\mu \nu}-\frac{1}{2 m^{2}} K_{\mu \nu}=0=T_{\mu \nu}
$$

This kind of black hole configurations were discussed in [24] and dubbed stealth. Note that this stealth solution corresponds to a particular case of the one found in [25].

We now show that the same field equations (2) may also accommodate Lifshitz black hole solutions for a different region of the parameters as it occurs in the source free case with the AdS solution [9] and the $z=3$ Lifshitz solution [10].

\section{ASYMPTOTICALLY LIFSHITZ BLACK HOLE SOLUTIONS}

There are three branches of solutions with Lifshitz asymptotic for the above equations of motion (2). In all of them the solutions have the following generic form

$$
\begin{aligned}
d s^{2} & =r^{2 z}\left(1-\frac{c_{1}}{r^{\chi}}\right) d t^{2}+\frac{d r^{2}}{r^{2}\left(1-\frac{c_{1}}{r^{\chi}}\right)}+r^{2} d \varphi^{2}, \\
\Phi(r) & =\frac{\sqrt{\alpha}}{r^{\chi / 2}},
\end{aligned}
$$

with the cosmological term and the graviton mass parameterized in term of the dynamical exponent as

$$
\Lambda=-\frac{1}{2}\left(z^{2}+z+1\right), \quad m^{2}=-\frac{1}{2}\left(z^{2}-3 z+1\right),
$$

where from now on we set $l=1$. The different branches share basically the same features than those discussed in the AdS case, and are presented with some detail below.

$$
\text { A. } \chi=(z+1)
$$

For $\chi=(z+1)$, there exists a Lifshitz black hole solution where the parameters are fixed as follows

$$
\begin{aligned}
\xi & =\frac{5}{32}, \quad \alpha=\frac{16 c_{1}(1-z)}{z^{2}-3 z+1}, \\
M & =\frac{1}{16}\left(z^{2}-3 z+1\right) \\
\lambda & =\frac{3\left(z^{2}-3 z+1\right)\left(8 z^{2}+11 z+13\right)}{256(z-1)} .
\end{aligned}
$$

In order to deal with a real scalar field, the constant $c_{1}$ must be strictly positive and the existence of a horizon is ensured for

$$
z \in\left[0, \frac{3-\sqrt{5}}{2}[\cup] 1, \frac{3+\sqrt{5}}{2}[.\right.
$$

As in the previous sections the other values at the boundaries of the intervals are excluded from this family of solutions.

$$
\text { B. } \chi=2(z-1)
$$

The second family of Lifshitz black hole solutions is only valid for $z \geq 1$ in order to have the correct Lifshitz asymptotic, and the parameters take the following form

$$
\begin{aligned}
\xi & =\frac{2 z-1}{4(3 z-1)}, \quad \alpha=\frac{4 c_{1}(1-3 z)}{z^{2}-3 z+1}, \\
M & =\frac{(2 z-5)\left(z^{2}-3 z+1\right)}{6 z-2}, \\
\lambda & =\frac{3\left(z^{2}-3 z+1\right)\left(12 z^{3}-44 z^{2}+48 z-13\right)}{4(1-3 z)^{2}} .
\end{aligned}
$$

As in previous case, in order to ensure a well behaved spacetime at infinity as well as having an event horizon, the dynamical exponent must belong to the following range

$$
z \in\left[1, \frac{3+\sqrt{5}}{2}[\text {. }\right.
$$

$$
\text { C. } \chi=\frac{1}{2}(z+1)
$$

The last class of solutions is given for $\chi=\frac{1}{2}(z+1)$ with

$$
\begin{aligned}
\xi & =\frac{3 z^{2}-4 z+3}{2\left(9 z^{2}-12 z+11\right)}, \\
\alpha & =\frac{c_{1}(z-3)\left(9 z^{2}-12 z-11\right)}{2(z-1)\left(z^{2}-3 z+1\right)}, \\
M & =\frac{(z-1)\left(21 z^{2}-13 z^{2}+31 z-15\right)}{16\left(9 z^{2}-12 z-11\right)} \\
\lambda & =-\frac{3(z-1)^{3}\left(z^{2}-3 z+1\right)\left(9 z^{2}-12 z-19\right)}{4(z-3)\left(9 z^{2}-12 z-11\right)^{2}} .
\end{aligned}
$$

The dynamical exponent falls within the following range

$$
z \in\left[0, \frac{3-\sqrt{5}}{2}[\cup] 1, \frac{3+\sqrt{5}}{2}[\cup] 3, \infty[.\right.
$$

In contrast with the previous cases, here there is wider range of possible values for the dynamical exponent. Note that none 
of these three classes of solutions accomodate a stealth with $z=3[10$ as it was the case for the asymptotically AdS solution. As a last comment we also notice that minimally coupled scalar fields are not allowed in the Lifshitz case.

\section{CONCLUSIONS}

In this note, we have reported two classes of black hole solutions of new massive gravity in three dimensions with a source described in term of a self-interacting and massive scalar field (non)minimally coupled. The first metric family corresponds to an asymptotically AdS black hole in a specific region of the coupling constants which are all parameterized in term of $\xi$. As in the free source case, we have shown that the same equations also admit Lifshitz black hole solutions for a different set of the parameters expressed in this case in term of the dynamical exponent $z$. It is somehow appealing that the equations of new massive gravity with or without source may accommodate such classes of black holes with different asymptotic behavior. It is also surprising that the matter source that has made possible the construction of these black hole solutions is quite simple. Indeed, it involves a scalar field $\Phi$ that can be massive, (non)minimally coupled and the self-interacting potential is a physical one $U \propto \Phi^{4}$. We have also computed the Wald formula for the entropy and realized that it is proportional to the lapse metric function evaluated at the horizon. As a consequence, the Wald entropy vanishes identically in spite of the fact that the solutions have a non zero temperature. We have not computed the mass since imposing that the first law of thermodynamics holds, this would yield to a zero mass. Indeed, in Ref. [19], there were obtained planar black hole solutions for Lovelock gravity with a scalar field nonminimally coupled which as well have zero entropy when computed using Wald's formula. In this case, using the Euclidean formalism, it was explicitly shown that the mass indeed vanishes. Independently, it will be nice to provide a complete thermodynamics analysis of the solutions here derived. Also it will be desirable to understand the physical meaning of these solutions which have a zero entropy. It seems that it is due to the presence of higher-order curvature terms as well as to the fact that the transverse section of these solutions is planar. The scalar field found here is static in both families of solutions, hence a rotating version of the AdS black hole solution can easily be obtained by operating with an improper boost in the $(t-\varphi)$-plane. This trick will also work in the case of the Lifshitz black hole with the difference that the spinning version of the Lifshitz black hole will violate the Lifshitz isometry at infinity. In three dimensions, solitons can easily be constructed from static black holes by operating a double Wick rotation. In the case of Lifshitz black holes with dynamical exponent $z$, the corresponding soliton will enjoy the Lifshitz anisotropy asymptotically with a dynamical exponent $z^{-1}$. These solitons may be useful to better understand the thermodynamics issue of the solutions presented here. Indeed, in Ref. [26], the authors proposed a generalization of the Cardy formula in order to compute the semiclassical entropy of Lifshitz black hole with dynamical exponent $z$, and in this formula, the ground state is played by the soliton with dynamical exponent $z^{-1}$.

\section{Acknowledgments}

FC is partially supported by grant 11121651 from FONDECYT and from CONICYT 79112034. MH is partially supported by grant 1130423 from FONDECYT and from CONICYT, Departamento de Relaciones Internacionales "Programa Regional MATHAMSUD 13 MATH-05". MH also acknowledge the CECs for his kind hospitality where part of this work has been done. JO is partially supported by grant 1141073 from FONDECYT. The Centro de Estudios Científicos (CECs) is funded by the Chilean government through the Centers of Excellence Base Financing Program of Conicyt.
[1] M. Bañados, C. Teitelboim and J. Zanelli, Phys. Rev. Lett. 69, 1849 (1992) hep-th/9204099.

[2] J. M. Maldacena, Adv. Theor. Math. Phys. 2, 231 (1998) hep-th/9711200.

[3] S. Deser, R. Jackiw and S. Templeton, Annals Phys. 140, 372 (1982) [Erratum-ibid. 185, 406 (1988)] [Annals Phys. 185, 406 (1988)] [Annals Phys. 281, 409 (2000)].

[4] E. A. Bergshoeff, O. Hohm and P. K. Townsend, Phys. Rev. Lett. 102, 201301 (2009) [arXiv:0901.1766][hep-th]].

[5] E. Ayon-Beato, G. Giribet and M. Hassaine, JHEP 0905, 029 (2009) arXiv:0904.0668 [hep-th]].

[6] G. Clement, Class. Quant. Grav. 26, 105015 (2009) arXiv:0902.4634 [hep-th]].

[7] H. Ahmedov and A. N. Aliev, Phys. Lett. B 694, 143 (2010) arXiv:1008.0303 [hep-th]].

[8] H. Ahmedov and A. N. Aliev, Phys. Rev. D 83, 084032 (2011) arXiv:1103.1086 [hep-th]].

[9] J. Oliva, D. Tempo and R. Troncoso, JHEP 0907, 011 (2009) arXiv:0905.1545 [hep-th]].

[10] E. Ayon-Beato, A. Garbarz, G. Giribet and M. Hassaine, Phys. Rev. D 80, 104029 (2009) arXiv:0909.1347 [hep- th]].

[11] S. A. Hartnoll, Class. Quant. Grav. 26 (2009) 224002 arXiv:0903.3246 [hep-th]].

[12] C. P. Herzog, J. Phys. A 42, 343001 (2009) arXiv:0904.1975 [hep-th]].

[13] S. Kachru, X. Liu and M. Mulligan, Phys. Rev. D 78, 106005 (2008) arXiv:0808.1725 [hep-th]].

[14] C. Martinez and J. Zanelli, Phys. Rev. D 54, 3830 (1996) gr-qc/9604021.

[15] M. Henneaux, C. Martinez, R. Troncoso and J. Zanelli, Phys. Rev. D 65, 104007 (2002) hep-th/0201170.

[16] F. Correa, C. Martinez and R. Troncoso, JHEP 1101, 034 (2011) arXiv:1010.1259 [hep-th]], JHEP 1202, 136 (2012) arXiv:1112.6198 [hep-th]].

[17] F. Correa, A. Faundez and C. Martinez, Phys. Rev. D 87, 027502 (2013) arXiv:1211.4878 [hep-th]], J. Aparicio, D. Grumiller, E. Lopez, I. Papadimitriou and S. Stricker, JHEP 1305, 128 (2013) arXiv:1212.3609 [hep-th]].

[18] W. Xu and L. Zhao, Phys. Rev. D 87, no. 12, 124008 (2013) arXiv:1305.5446 [gr-qc]].

[19] F. Correa and M. Hassaine, JHEP 1402, 014 (2014) 
arXiv:1312.4516 [hep-th]].

[20] J. D. Bekenstein, Annals Phys. 82, 535 (1974).

[21] N. M. Bocharova, K. A. Bronnikov and V. N. Melnikov, Vestn. Mosk. Univ. Fiz. Astron. 6 (1970) 706 [Moscow Univ. Phys. Bull. 25 (1970) 80].

[22] C. Martinez, R. Troncoso and J. Zanelli, Phys. Rev. D 67, 024008 (2003) arXiv:hep-th/0205319.

[23] E. A. Bergshoeff, O. Hohm and P. K. Townsend, Phys.
Rev. D 79, 124042 (2009) arXiv:0905.1259 [hep-th]].

[24] E. Ayon-Beato, C. Martinez and J. Zanelli, Gen. Rel. Grav. 38, 145 (2006) hep-th/0403228.

[25] M. Hassaine, Phys. Rev. D 89, 044009 (2014) arXiv:1311.4623 [hep-th].

[26] H. A. Gonzalez, D. Tempo and R. Troncoso, JHEP 1111, 066 (2011) arXiv:1107.3647 [hep-th]]. 